\title{
CHALLENGES WITH UNUSUAL INFECTION OF UNSUAL SEVERITY - METHICILLIN RESISTANT STAPHYLOCOCCUS AUREUS INFECTION
}

B. Shenoy ${ }^{1}$, Shivakumar . $\mathrm{S}^{2}$, Ashwath Ram . $\mathrm{R}^{3}, \mathrm{~B}$.Bharath ${ }^{4}$. ${ }^{1}$ Head of Paediatric Infectious Diseases $,{ }^{2},{ }^{3}$ Paediatric Intensivists

${ }^{4}$ Fellow in Paediatric Infectious Diseases.

Manipal Hospital , Bangalore, India.

\section{INTRODUCTION}

MRSA is an important cause of morbidity \& mortality. Recently CA-MRSA infections are on the rise. Hence, a strong index of suspicion is important to diagnose and treat early.

\section{CASE HISTORY}

A4 yr old child presented with complaints of

* Pain in the right shoulder for 6 days

* Followed by pain in the hip which was later followed by generalized body pain

- Fever for 4 days.

Child was toxic, febrile and unable to walk and move his right hand. He had generalized swelling of the above limbs which were tender.

\section{INVESTIGATION}

* Blood parameters showed leukopenia, thrombocytopenia, high CRP $(237 \mathrm{mg} / \mathrm{dl})$.

- MRI of the right upper limb showed features of osteomyelitis in the right humerus and consolidation involving the right lung.

* Whole body MRI was done which additionally showed myositis of both thigh muscles

- CT chest confirmed bilateral lung consolidation.

* Blood culture grew MRSA.

\section{MANAGEMENT}

* Child was treated with vancomycin and linezolid.

* Child was intubated on day 4 of admission post surgery (Curettage, Saucerisation).

* Nearly $200 \mathrm{ml}$ pus was drained from the right upper arm. Right chest drain was also inserted.

- A course of IVIG was given for persistent toxemic symptoms

\section{DISCUSSION}

Invasive MRSA infections associated with the production of PVL toxins have high mortality despite adequate treatment.

This case is presented to discuss

* Issues with CA-MRSA infections, clinical clues to early recognition and appropriate treatment.

* When to suspect infections due to strains producing PVL toxin.

* Empirical choice of antibiotics

* Interpreting cultures of MRSA bacteremia

* Role of various antimicrobials and IVIG.

- Ideal investigation and treatment for osteomyelitis,

* Community level prevention strategies and

* Carrier detection issue with MRSA infections.

\section{KEYWORDS}

MRSA, Osteomyelitis, Pneumonia 